\title{
ON THE ACCELERATION AND JERK IN MOTION ALONG A SPACE CURVE WITH QUASI-FRAME IN EUCLIDEAN 3-SPACE
}

\author{
Ayman Elsharkawy ${ }^{1}$ and Ahmed Elshenhab ${ }^{2}$ \\ ${ }^{1}$ Faculty of science, tanta university \\ ${ }^{2}$ Mansoura University Faculty of Science
}

October 26, 2020

\begin{abstract}
In this paper, we consider a particle moves on a space curve in the Euclidean 3-space and resolve its acceleration and jerk vectors according to quasi-frame. In this resolution, by appyling Siacci's theorem, we state the acceleration vector as the sum of its tangential and radial components, and obtain the jerk vector along the tangential direction and radial directions in osculating and rectifying planes. On the basis of the jerk vector formula, we give the maximum admissible speed on a space curve at all trajectory points. Furthermore, we present illustrative examples to explain how our results work.
\end{abstract}

\section{Hosted file}

On the Acceleration and Jerk in motion along a space curve.pdf available at https: //authorea.com/users/347554/articles/488998-on-the-acceleration-and-jerk-in-motionalong-a-space-curve-with-quasi-frame-in-euclidean-3-space 